\title{
Design and Performance Evaluation of QPSK Modulation and Demodulation in SS mode Based on Systemview
}

\author{
Sheng LIANG ${ }^{a}$, Gaofeng PAN ${ }^{b}$, Youxing WU, Yong XIE \\ China Satellite Maritime Tracking and Control Department, Jiangyin, Jiangsu, 214431,China \\ a murongxd@163.com, b pgfzhy@163.com, c emi91@sina.com
}

Keywords: SS; QPSK; Eye Diagram.

Abstract. Because the modulation and demodulation method in SS (Spread Spectrum) mode is different with sub-carrier TT\&C system, so it is necessary to realize its performance evaluation based on software simulation. This paper makes use of Systemview to simulate the single target measurement in SS system and evaluate its anti-noise performance by eye diagram and BER. Results show the simulation model designed in this paper is feasible and Systemview can visualize abstract communication simulation.

\section{Introduction}

The present SS TT\&C system has two kinds, which are coherent and non-coherent SS modes. In coherent mode, telecommand message is embedded in branch I of QPSK modulation after encoded with short PN code (period $2^{10}-1$ ), distance measure message is embedded in branch $\mathrm{Q}$ after encoded with long PN code (period $\left.\left(2^{10}-1\right) \times 256\right)$ and the two branch signals with different powers compose UQPSK signal. In non-coherent mode, two branch messages encoded with short PN code are BPSK modulated respectively, which will be transmitted to the satellite after up-conversion and power amplification. This paper sets conditions to unitize the two modes and designs the modulation \& demodulation simulation model based on Systemview to evaluate performance of SS mode.

\section{Design Frame of System Principle}

After setting the two BPSK signals to use orthogonal carrier modulation, two modes are unified together (That is QPSK). It not only simplifies the design frame of system principle, but also doesn't affect the simulation of signal processing flow and anti-jamming performance evaluation.

Fig. 1 shows the frame of system principle designed in this paper.

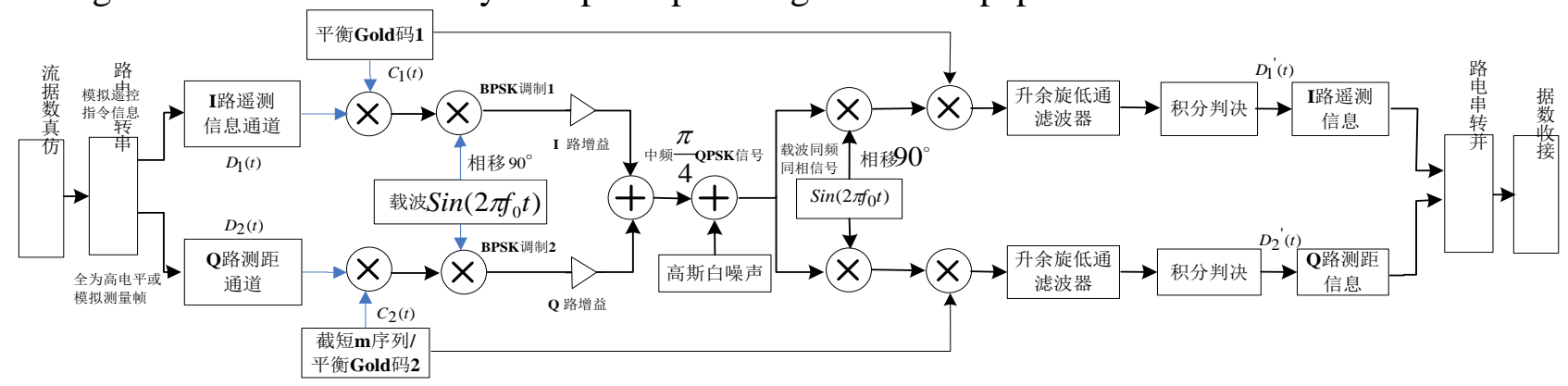

Fig. 1 Frame of system principle

By Fig. 1, orthogonal modulation and coherent demodulation are used in QPSK ${ }^{[1]}$ and branch Q can chooses Modified de Bruijn sequences or balanced Gold code to be encoded. Keeping in mind that in order to acquire low-pass character and reduce ISI (Inter-symbol-interference), raised cosine filter is adopted in receive link ${ }^{[2]}$. Key parameters are set as follows:

1) Bit rate of branch I \& Q data : 1Mbps;

2) Bit rate of SS code: $5 \mathrm{Mbps}$;

3) Frequency of carrier: 70MHz;

4) Power ratio of branch I \& Q: 1. 


\section{Modulation and Demodulation Design of QPSK in SS Mode Based on Systemview}

Fig. 2 has given the simulation model of QPSK modulation \& demodulation based on Systemview in line accordance with the principle frame.

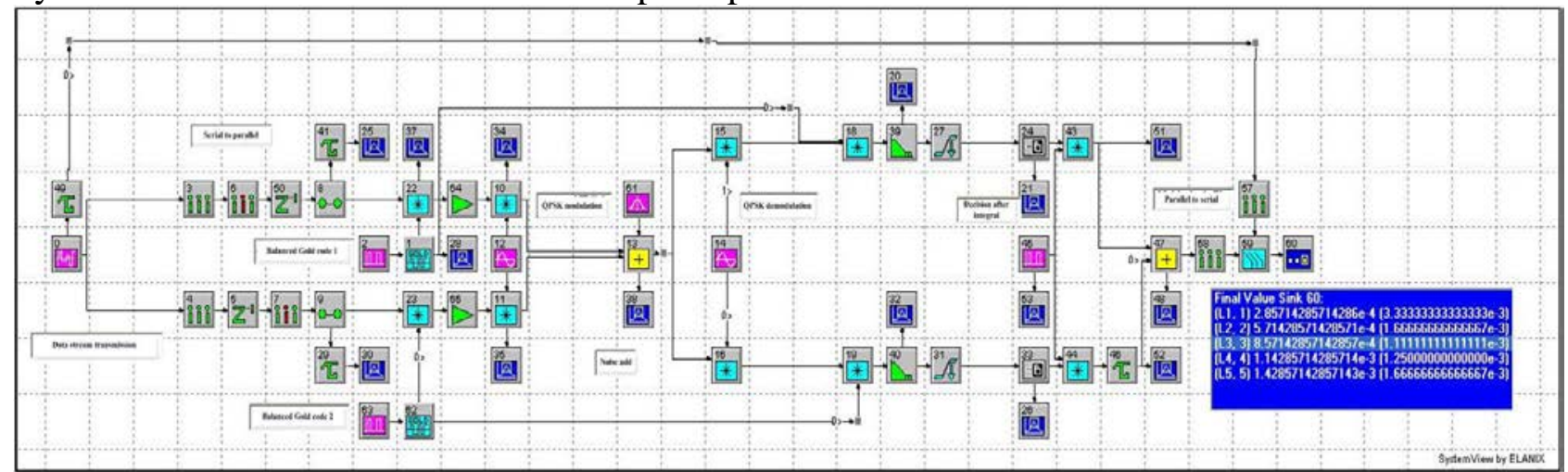

Fig. 2 Simulation model of QPSK modulation \& demodulation based on Systemview

As Fig. 2, from icon 13, the head part accomplishes QPSK modulation and in the meantime, the latter part realizes demodulation and integral decision; Icon 61 acts as Gaussian Noise generator; icon 12 and 14 act as sine wave generator. Two orthogonal BPSK signals add together after data stream multiplied with carrier to get UQPSK signal (Power adjusting by icon 64 and 65). The bottom right corner of Fig. 2 shows calculated values of BER.

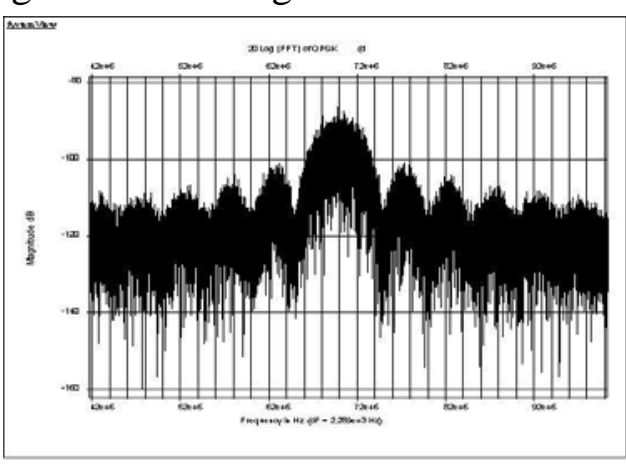

(a) Frequency spectrogram of QPSK IF

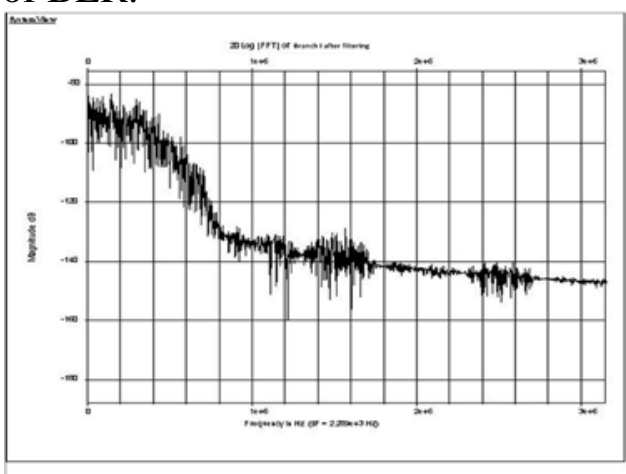

(b) Frequency spectrogram of branch I after filtering

Fig. 3 Frequency spectrogram of simulation signal

Fig. 3(a) shows the spectrogram of QPSK IF with Gaussian noise added from icon 38, $70 \mathrm{MHz}$ is the center frequency and main lobe width is $10 \mathrm{MHz}$, it has proved simulation results accord with key parameters' demand. Fig. 3(b) shows the spectrogram of branch I after raising cosine filtering, IF moves to base frequency and real main lobe width after despreading changes to $1 \mathrm{MHz}$. Follow sections will introduce PN code generator model and serial-to-parallel model.

Structure and Generating of Balanced Gold Code. Common PN codes mainly have Modified de Bruijn sequences and balanced Gold code. Between the two codes, self-correlation and cross-correlation of Gold code are better, further more, no of addresses is bigger. Order 5 balanced Gold code generator shown in Fig. 4(a) is made up by order 5 preferred pair [67] $]_{8}$ and $[45]_{8}$, whose Systemview simulation model can be found in Fig. 4(b).

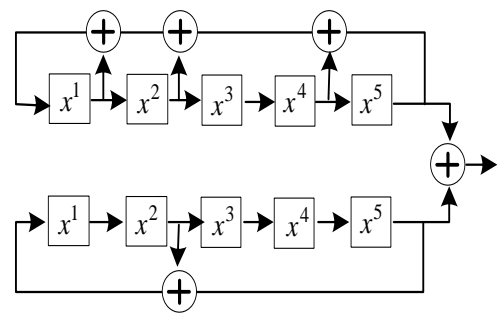

(a) Schematic diagram

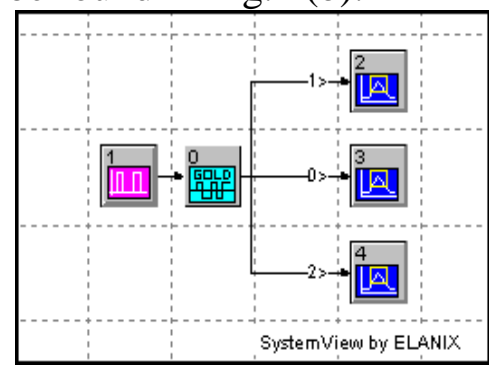

(b) Simulation model

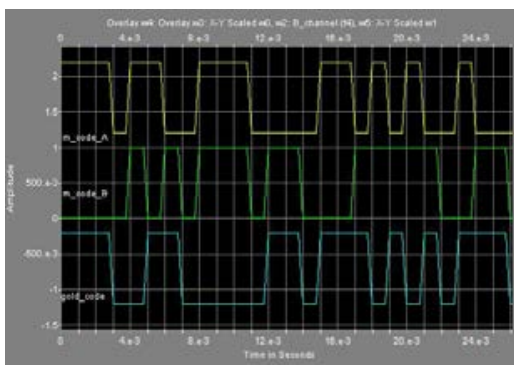

(c) Simulation result

Fig. 4 Generating and simulation of balanced Gold Code based on Systemview 
In Fig. 4(b), icon 0 is used to generate Gold code by setting parameters. Firstly, we write the taps of preferred pairs, these are $(1,2,4,5)$ and $(2,5)$; Secondly, set initial values as $(1,3,4,5)$ and (1); in the end, chose " 0,1 " as output format to generate Gold code shown in Fig. 4(c) (The above two show preferred pairs and the bottom shows designed Gold code).

Design of Serial-to-parallel Model. Serial-to-parallel circuit can divide data stream to branch I and Q. From Fig. 5(a), the same data streams are sampling at $1 \mathrm{MHz}$ and by adding delay unit(icon 73 and 68), odd and even codes output separately. Simulation result in Fig. 5(b) shows the model is correct and feasible.

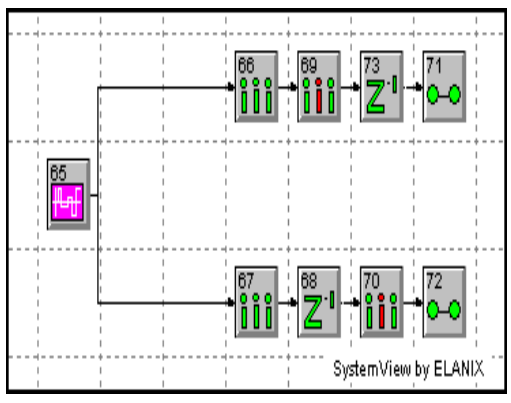

(a) Serial-to-parallel model

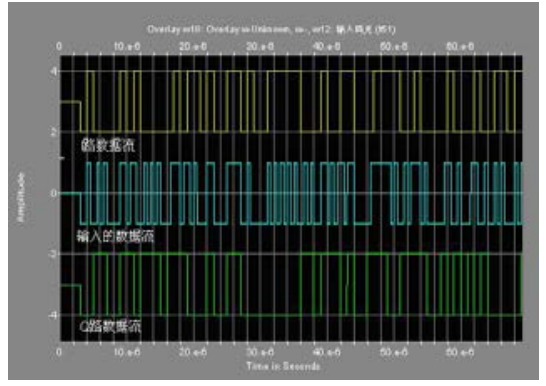

(b) Simulation result

Fig. 5 Design and simulation of Serial-to-parallel circuit based on Systemview

Fig. 6(a) gives the design of parallel-to-Serial model. We resample data with $1 \mathrm{MHz}$, duty-cycle 0.5 square wave to realize parallel-to-Serial. Fig.6(b) show the correct simulation result.

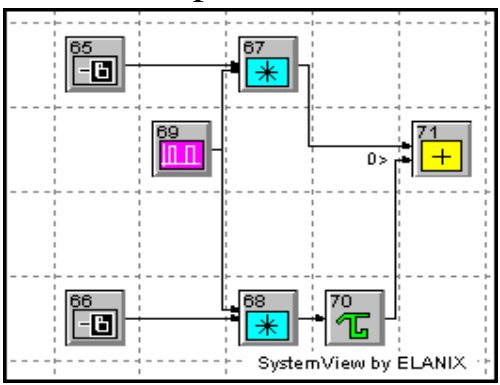

(a) parallel-to-Serial model

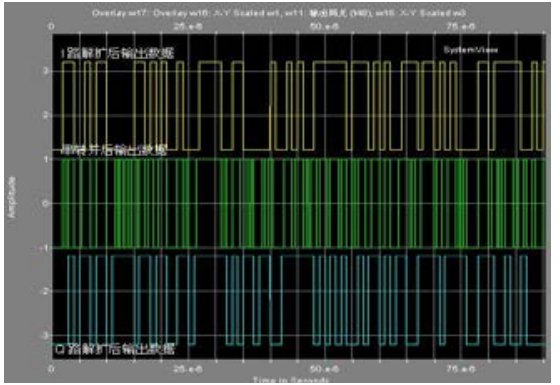

(b) Simulation result

Fig. 6 Design and simulation of parallel-to-Serial circuit based on Systemview

\section{Simulation for System Anti-noise Performance}

ISI leads to performance degradation in SS TT\&C system. In reality, eye pattern can be used to evaluate system anti-noise performance qualitatively, which is one kind of baseband waveform ${ }^{[3]}$. Change about eye pattern's character is closely related with BER. Serious ISI or strong channel noise will result in "closed eye" and system can't work.

According to the relationship between jamming strength and eye pattern's character, we increase the variance of icon 61 in Fig. 2 step by step and get the mean value for BER by lots of simulations, as Fig. 7 shown. 


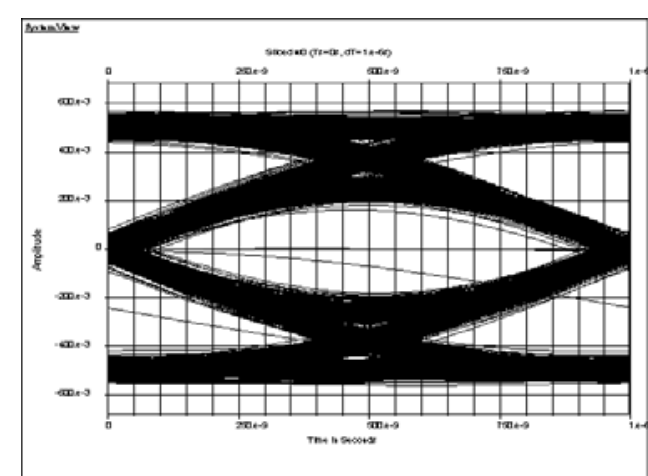

(a) $\mathrm{BER}=2.778 \times 10^{-4}$

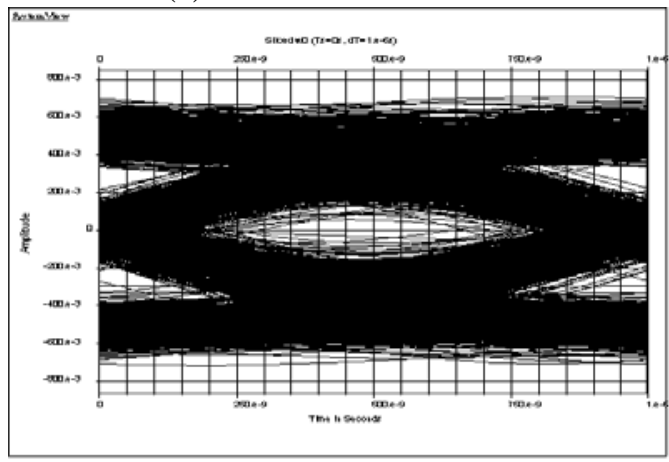

(a) $\mathrm{BER}=2.056 \times 10^{-2}$

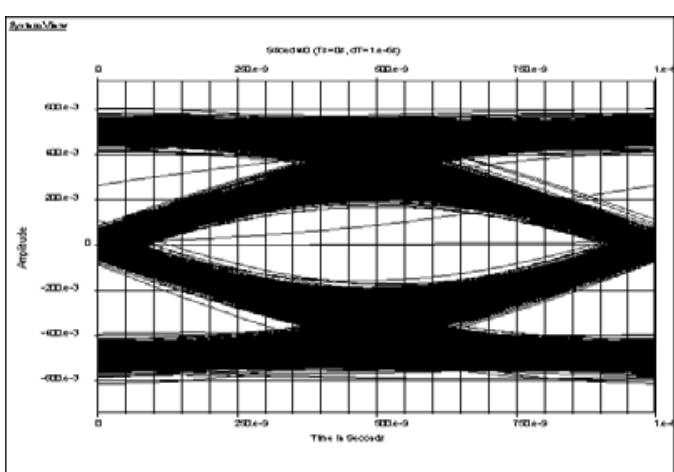

(b) $\mathrm{BER}=1.944 \times 10^{-3}$

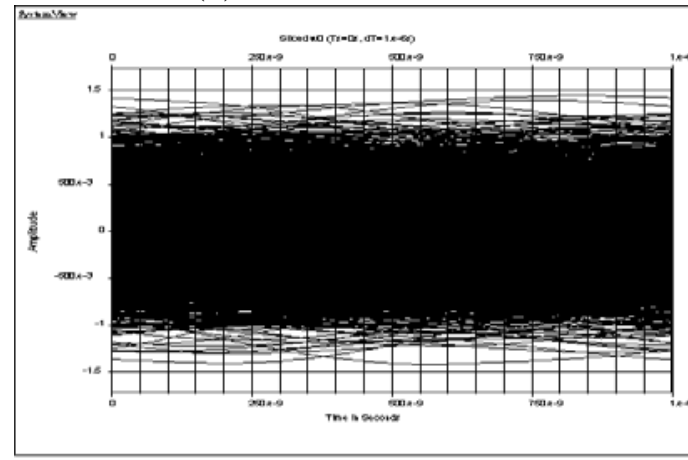

(b) $\mathrm{BER}=1.528 \times 10^{-1}$

Fig. 7 Relationship between BER and eye patterns

From Fig. 7, we find that eye pattern can directly reflect severity of noise jamming, eyelid shows sampling distortion and eye size reflects noise tolerance.

\section{Conclusions}

This paper designs the simulation model for QPSK modulation \& demodulation in SS mode based on Systemview including signal generating, encoding, modulation, noise adding, demodulation, filtering and decision. Eye pattern is compared with BER to evaluate system anti-noise performance qualitatively. Simulation results show principle frame and model design are correct and feasible.

\section{References}

[1] SHEN Yunchun. Spread Spectrum Technique[M]. Beijing: Defense Industry Press, 1995. (in Chinese)

[2] OUYANG Changyue. Information Transmission Foundation[M]. Beijing: Beijing University of Aeronautics and Astronautics Press, 1995. (in Chinese).

[3] QING Song, Chen Daisong, WU Jianhua. Systemview Simulation \& Analysis about Digital Communication System[M]. Beijing: Beijing University of Aeronautics and Astronautics Press, 2001. (in Chinese). 